Ioannis Prassas, Clare Fiala and Eleftherios P. Diamandis*

\title{
Assay requirements for COVID-19 testing: serology vs. rapid antigen tests
}

https://doi.org/10.1515/cclm-2021-0234

Received January 28, 2021; accepted March 11, 2021;

published online March 18, 2021

Keywords: COV-2; COVID; SARS; serology.

To the Editor,

Apart from the molecular diagnostic PCR test (gold standard), there are two other types of SARS-COV-2-related tests that are fundamental for our battle against the COVID-19 pandemic:

a. Serological tests measure host antibodies against SARS-COV-2 to delineate possible past infection. These tests can also be used to assess disease prevalence and monitor the dynamics of individual immunological responses over time [1].

b. Rapid antigen tests measure SARS-COV-2 proteins to determine the putative COVID-19 contagiousness state. The usefulness of frequent COVID-19 antigen testing through inexpensive, simple and rapid tests has been established [2]. These tests can contribute tremendously to COVID-19 infection control, even if their analytical sensitivity is two to three orders of magnitude lower than the benchmark PCR test [2].

The boundaries of intended utility for both of these tests are heavily dictated by their analytical and clinical limitations.

*Corresponding author: Eleftherios P. Diamandis, MD, PhD, FRCP(C), FRSC, Head of Clinical Biochemistry, Mount Sinai Hospital and University Health Network, 60 Murray St. Box 32, Floor 6, Rm L6-201, Toronto, ON, M5T 3L9, Canada; Department of Pathology and Laboratory Medicine, Mount Sinai Hospital, Toronto, ON, Canada; Department of Laboratory Medicine and Pathobiology, University of Toronto, Toronto, ON, Canada; and Department of Clinical Biochemistry, University Health Network, Toronto, ON, Canada, Phone: +416 586 8443,

E-mail: Eleftherios.diamandis@sinaihealth.ca. https://orcid.org/ 0000-0002-1589-820X

Ioannis Prassas and Clare Fiala, Department of Pathology and Laboratory Medicine, Mount Sinai Hospital, Toronto, ON, Canada
Here, we draw attention to the reality that the consequences of false positive (FP) or false negative (FN) results using these complementary tests are diametrically different. With serology, a FP result is much more consequential than a FN result. A FP can falsely reassure individuals that they have already been infected and are presumably immune, thus prompting them to return to their regular activities without the need for strict protective measures. Thus, serology FP patients are potential spreaders. Conversely, with antigen testing, the FN results are potentially much more consequential than the FP ones since a patient with a FN antigen test may be considered as a non-spreader. Similar to the FN serology patients, the FP patients of antigen tests may be unduly quarantined or advised to follow strict mitigation measures, but they cannot spread the virus.

As with any other medical laboratory testing, it is also important to consider the positive and negative predictive values (PPV, NPV). It is well-known that these parameters are highly dependent on disease prevalence. PPV represents the patient's chance of having the disease if the test is positive while NPV represents the patient's chance of not having the disease if the test is negative [3].

In Table 1, we compiled some hypothetical data which are representative of the sensitivities and specificities of antigen and serological tests for COVID-19 and its prevalence in various countries/regions [4-6]. The following comments apply for the mentioned assumptions.

(1) The NPV of both antigen and serology tests is always very high, if the assumed prevalence is $20 \%$ or less. Consequently, a negative test of either type (antigen or serology) suggests ruling out the disease with high confidence $(>97.5 \%)$.

(2) The PPV surpasses 90\% (a high-confidence rule-in result) only when the prevalence is $>15 \%$ and the test's specificity surpasses $99 \%$. At a lower prevalence or a lower test specificity, the PPV is lower; in many cases $<50 \%$. The test's sensitivity has a relatively minor effect on the PPV.

(3) For serological tests, where FP patients can spread the disease, the numbers of FP patients range from 800 to 5,000 per 100,000 individuals (as per Table 1 assumptions). The major determinant is assay specificity, not 
Table 1: Diagnostic test characteristics at varying scenarios of prevalence, sensitivity and specificity ${ }^{\mathrm{a}}$.

\begin{tabular}{|c|c|c|c|c|c|c|}
\hline & & & $\mathrm{FP} / \mathbf{1 0 0 , 0 0 0}$ & $\mathrm{FN} / \mathbf{1 0 0 , 0 0 0}$ & PPV & NPV \\
\hline Prevalence & & & & & & $1 \%$ \\
\hline Sensitivity/specificity & 90 & 95 & 4,950 & 100 & 15.4 & 99.9 \\
\hline Sensitivity/specificity & 90 & 99 & 990 & 100 & 47.6 & 99.9 \\
\hline Sensitivity/specificity & 98 & 95 & 4,950 & 20 & 16.6 & 99.9 \\
\hline Sensitivity/specificity & 98 & 99 & 990 & 20 & 49.7 & 99.9 \\
\hline Prevalence & & & & & & $2 \%$ \\
\hline Sensitivity/specificity & 90 & 95 & 4,900 & 200 & 26.9 & 99.8 \\
\hline Sensitivity/specificity & 90 & 99 & 980 & 200 & 64.7 & 99.9 \\
\hline Sensitivity/specificity & 98 & 95 & 4,900 & 40 & 28.6 & 99.9 \\
\hline Sensitivity/specificity & 98 & 99 & 980 & 40 & 66.7 & 99.9 \\
\hline \multicolumn{7}{|l|}{ Prevalence } \\
\hline Sensitivity/specificity & 90 & 95 & 4,750 & 500 & 48.6 & 99.4 \\
\hline Sensitivity/specificity & 90 & 99 & 950 & 500 & 82.6 & 99.4 \\
\hline Sensitivity/specificity & 98 & 95 & 4,750 & 100 & 50.8 & 99.9 \\
\hline Sensitivity/specificity & 98 & 99 & 950 & 100 & 83.8 & 99.9 \\
\hline Prevalence & & & & & & $10 \%$ \\
\hline Sensitivity/specificity & 90 & 95 & 4,500 & 1,000 & 66.7 & 98.8 \\
\hline Sensitivity/specificity & 90 & 99 & 900 & 1,000 & 90.9 & 98.9 \\
\hline Sensitivity/specificity & 98 & 95 & 4,500 & 200 & 68.5 & 99.8 \\
\hline Sensitivity/specificity & 98 & 99 & 900 & 200 & 91.6 & 99.8 \\
\hline Prevalence & & & & & & $20 \%$ \\
\hline Sensitivity/specificity & 90 & 95 & 4,000 & 2000 & 81.8 & 97.4 \\
\hline Sensitivity/specificity & 90 & 99 & 800 & 2000 & 95.7 & 97.5 \\
\hline Sensitivity/specificity & 98 & 95 & 4,000 & 400 & 83.1 & 99.5 \\
\hline Sensitivity/specificity & 98 & 99 & 800 & 400 & 96.1 & 99.5 \\
\hline
\end{tabular}

${ }^{a}$ Numbers refer to patients per 100,000 hypothetical population. FP, false positive; FN, false negative; PPV, positive predictive value; NPV, negative predictive value. Numbers of true positives and true negatives are not shown. Sensitivity and specificity are expressed as percentage (\%). For more details and discussion see text.

prevalence or sensitivity. Serology tests should be designed to maximize specificity in order to minimize FP.

(4) For antigen tests, where FN patients can spread the disease, the numbers of FN range from 20 to 2,000 per 100,000 individuals (as per Table 1 assumptions) with the major determinant of FN patients being prevalence and assay sensitivity. Lowest FN numbers are seen with low disease prevalence and high assay sensitivity, not specificity. This means that antigen tests should be designed to maximize sensitivity, in order to minimize FN.

We recommend that manufacturers of COVID-19 tests maximize specificity with serology and sensitivity with antigen/nucleic acid tests to minimize the number of potential disease spreaders who are either FP or FN, respectively.

Looking forward: PCR-based and viral antigen-based tests in nasopharyngeal swabs or saliva (rapid, point of care, or otherwise) will continue to be used widely for many months to years, since they are the most reliable means of establishing active infection. The results of these diagnostic tests are actionable through isolation and therapeutic measures [2]. The serological tests are currently used only in surveillance and seroconversion studies and have no immediately actionable consequences [1]. However, in the ensuing 6-12 months, we predict these tests' utility to increase in order to study quantitative levels of antibodies in both naturally infected and vaccinated individuals. In such cases, serological assays may yield actionable results in terms of deciding who and when should be revaccinated. In short, our view is that both types of tests (diagnostic/antigen and serological) will soon be used hand-in hand to optimize the available preventative and therapeutic strategies for COVID-19 disease. In this case, our guidance of optimizing specificity and sensitivity of each test type, as mentioned above, should be considered.

Research funding: None declared.

Author contributions: All authors have accepted responsibility for the entire content of this manuscript and approved its submission.

Competing interests: Authors state no conflict of interest.

\section{References}

1. Whitman JD, Hiatt J, Mowery CT, Shy BR, Yu R, Yamamoto RN, et al. Evaluation of SARS-CoV-2 serology assays reveals a range of test performance. Nat Biotechnol 2020;38:1174-83. 
2. Mina MJ, Parker R, Larremore DB. Rethinking Covid-19 test sensitivity: a strategy for containment. N Engl J Med 2020;383:e120.

3. Diamandis P, Prassas I, Diamandis EP. Antibody tests for COVID-19: drawing attention to the importance of analytical specificity. Clin Chem Lab Med 2020;58:1144-5.

4. Figueiredo-Campos P, Blankenhaus B, Mota C, Gomes A, Serrno M, Ariotti $S$, et al. Seroprevalence of anti-SARS-CoV-2 antibodies in COVID-19 patients and healthy volunteers up to six months post disease onset. Eur J Immunol 2020;50:2025-40.
5. Borges LP, Martins AF, de Melo MS, Brito de Oliveria MK, Neto JMR, Dosea MB, et al. Seroprevalence of SARS-CoV-2 IgM and IgG antibodies in an asymptomatic population in Sergipe, Brazil. Rev Panam Salud Públic 2020;44:e108.

6. Manthei DM, Whalen JF, Schroeder LF, Sinay AM, Li S, Valdez R, et al. Differences in performance characteristics among four high throughput assays for the detection of antibodies against SARS-CoV-2 using a common set of patient samples. Am J Clin Pathol 2020:aqaa200. 